\title{
pathologie des silos
}

\author{
pathology of silos
}

\author{
D. AVERSENC \\ Président Directeur Général de la SOPENA SA*
}

Rev. Franç. Géotech. n 52, pp. 5-9 (juillet 1990)

\section{Résumé}

Les silos ont pour but de stocker les grains et autres matières pondérales, et de les abriter des intempéries.

On rencontre le plus souvent des désordres mettant en cause l'étanchéité des parois : infiltration d'eau au droit de la galerie enterrée, gênant le transfert des grains par tapis roulant, ou passage d'eau au travers de la paroi, mouillant le grain stocké.

On rencontre également des fissures, entraînant ou non des infiltrations d'eau, causées par des insuffisances d'armatures, pouvant conduire à des ruptures.

Enfin, on rencontre des désordres consécutifs à un tassement du dallage et des fondations.

Tous ces désordres sont à apprécier, en fonction de l'usage du silo, et de la gêne qui s'ensuit pour son exploitation.

\section{Abstract}

Silos are used to store the grain and to shelter it from the bad weather.

Most often we meet with disorders questioning the water tightness of the walls : infiltration of water near the buried gallery, hindering the transfer of the grain by travelling band, or passage of water through the walls wetting the stocked grain.

Cracks are also met with, leading wheter to water infiltrations or not, caused by insufficient reinforcement which may lead to ruptures.

Finally disorders consecutive to a firming of the pavement and of the foundations are met with.

All these disorders are to be estimated according to the use of the silo and of the trouble which follows concerning its exploitation. 


\section{PRÉAMBULE}

La construction de silo est généralement considérée par les assureurs comme un risque important pour les entreprises et bureaux d'études, du fait des désordres qui souvent affectent ces ouvrages au cours de leur vie, et principalement pendant la période de garantie décennale.

Les risques consécutifs à la conception-réalisation de silos, résultent indiscutablement et pour une grand part d'une certaine méconnaissance du comportement des matières ensilées ne permettant pas d'approcher les valeurs réelles par les calculs d'une façon aussi précise que pour d'autres techniques telles que par exemple le béton armé ou la charpente métallique, et pour une autre part de limportance des efforts qui les sollicitent.

En ce qui concerne le droit, pendant de nombreuses années, les silos ont été considérés comme des ouvrages de génie civil, et les décisions de jurisprudence ont été relativement divergentes considérant tantôt que la garantie décennale était de droit, tantôt qu'elle ne l'était pas. Depuis maintenant 20 années, on peut considérer que d'une façon systématique les tribunaux considèrent que ces ouvrages relèvent de la garantie décennale due par les constructeurs et en conséquence des articles 1792 et 2270 du Code civil.

La loi du 4 janvier 1978 fait référence à la notion d'impropriété à destination : on peut considérer que deux natures de désordres sont susceptibles de rendre un silo impropre à sa destination :

- d'une part, des désordres de structure, compromettant ou susceptibles de compromettre d'une façon certaine, à terme, la stabilité de l'ouvrage ;

- d'autre part, des infiltrations d'eau ou de l'humidité susceptibles d'endommager les matières ensilées.

Des règles professionnelles, établies successivement, ont tenté de mettre de l'ordre dans la classification des types de silos, de définir les caractéristiques mécaniques des matières, enfin de définir les principes de calcul de ces ouvrages. En l'état actuel, ces règles sont relativement précises, pour certains types de silos, de magasins de stockage ou de silos réservoirs, mais sont muettes pour d'autres ouvrages à forme particulière, aucune des méthodes ne leur étant applicable. Par ailleurs, il faut bien dire, qu'il demeure une méconnaissance certaine, relative aux caractéristiques des matières ensilées en particulier leur hétérogénéité, leur cohésion, ainsi que les effets de la vidange. Il paraît étrange, qu'on prévoit un angle de talus supérieur à l'angle de frottement interne de la matière sans pour autant prendre en compte une quelconque cohésion.

Il serait intéressant d'analyser sommairement les principaux désordres affectant les silos, et relatifs à des problèmes de structure essentiellement.

\section{LES FONDATIONS}

Il est moins fréquent que les silos présentent une pathologie caractérisée des fondations. La raison en est vraisemblablement que compte tenu de l'investissement important et de la nature technique des silos, le maître d'ouvrage et les constructeurs n'hésitent pas à engager des frais relativement conséquents en vue d'une investigation des sols préalable à la construction et permettant de limiter les risques à ce titre, cout faible toutefois en comparaison du cout global de l'ouvrage.

\section{INFILTRATIONS DANS LES GALERIES}

\section{Ce désordre est au contraire très répandu.}

Le premier problème qui se pose est de savoir si ces infiltrations dans des galeries utilisées en vue de la manutention du grain sont de la nature des désordres qui rendent l'ouvrage impropre à sa destination. Il semble que les tâches d'humidité ou même quelques venues d'eau, en fond d'une galerie, ne gênent pas l'exploitation ; il en est évidemment à l'inverse s'il s'agit de venues intempestives ou d'eau stagnante.

L'autre problème est relatif au risque qu'a accepté ou non le maître de louvrage; en l'absence de documents spécifiques, il est évident que les constructeurs risquent devoir une étanchéité, ou à défaut un système de collecte des eaux avec pompe de relevage, à défaut que ces problèmes aient été explicités auprès du maître de l'ouvrage au moment de la construction, et par écrit, afin que celui-ci ait pu prendre sa décision en pleine connaissance.

Il faut donc que soient bien définies, en accord avec le maître de l'ouvrage, les dispositions d'étanchéité ou de collecte des eaux dans les parties enterrées, et que le risque qu'a accepté le maître d'ouvrage, moyennant une économie souvent substantielle, soit clairement explicité par un courrier autre que le CCTP, ce dernier n'étant qu'une pièce contractuelle entre le maître d'ouvrage et l'entreprise.

Les causes de venues d'eau, dans les parties enterrées, ont souvent les origines suivantes:

- des venues d'eau intempestives : en général ces venues d'eau n'ont pas pour origine une nappe phréatique, mais simplement le fait de l'eau météorique, qui tombe au pourtour du silo, pénètre dans la ou les couches superficielles relativement perméables et sont arrêtées par une couche plus profonde imperméable. A loccasion de l'étude de sol, il n'a pas été rencontré de nappe phréatique et aucun disposition particulière n'a donc été mise en place. Il n'empêche qu'à l'occasion de fortes pluies, l'eau se rassemble au pourtour de la galerie, et arrêtée par la couche semiprofonde imperméable, pénètre dans la galerie. C'est une nappe suspendue saisionnière ;

- une deuxième cause, qu'il y ait étanchéité, imperméabilisation ou aucun traitement particulier, provient 
bien évidemment des conditions de réalisation à savoir les reprises de bétonnage, ainsi que la microfissuration ou la porosité du béton, qui laisse passer l'eau ; - une troisième cause, constatée dans de nombreux cas, est la suivante: les règlements BA 68 prévoient dans nos régions des joints de dilatation tous les $35 \mathrm{~m}$ environ, un peu moins dans le Sud, un peu plus dans le Nord-Ouest et l'Ouest, mais précisent d'une façon d'ailleurs quelque peu ambiguë que des joints de dilatation ne sont pas nécessaires dans les parties enterrées. En fait, lorsque le silo est en cours d'exploitation, et pour une galerie par exemple de $100 \mathrm{~m}$ de longueur, compte tenu de la chaleur dégagée par le grain, et les appareils de manutention, compte tenu que l'hygrométrie est variable dans ces galeries, il est bien évident qu'on ne peut considérer la galerie comme un ouvrage enterré au sens du BA 68 ou BAEL à température et hygrométrie constantes, et qu'il faut des joints, qu'on les appelle de dilatation ou de retrait. Le problème est exactement similaire à celui des parkings enterrés soumis à une forte ventilation et à une forte déshydratation du béton. Nous avons constaté sur de nombreux silos dont la longueur des galeries dépassaient 30 ou $40 \mathrm{~m}$, une multitude de fissurations plus ou moins importantes correspondant indiscutablement à des phénomènes de retrait.

En tout état de cause, s'il est fait application d'un enduit étanche rigide à base de produit hydraulique, il est impératif de ne pas considérer une galerie enterrée comme un ouvrage enterré au sens du règlement BA 68 .

\section{LES SILOS TUBES}

C'est la pathologie traditionnelle des silos, qui consiste en l'apparition de micro-fissurations ou fissurations verticales au droit des trous laissés par les tiges de vérins des coffrages glissants (fig. 1). Bien entendu, il s'agit d'un point faible de la structure, même si théoriquement il ne devrait pas en être ainsi puisque le béton est considéré comme n'ayant pas de résistance à la traction.

Cette pathologie n'est généralement pas dangereuse et il peut y être remédié par l'application d'une étanchéité évitant la corrosion des armatures, et les infiltrations mouillant le grain stocké.

Les cas graves de fissuration verticales au droit des trous laissés par les tiges de vérins résultent généralement d'une mauvaise mise en œuvre à savoir des espacements sensiblement plus importants que ceux nécessaires ou prévus au plan, et aggravés par des calculs trop optimistes.

On peut se demander, quel est l'intérêt du rebouchage par injection des trous de tiges de vérins : d'une part subsistent fréquemment dans les trous, des tiges abandonnées et comme l'injection doit se faire de bas en haut, on voit mal comment on peut reboucher valablement ces trous, d'autre part, l'aspect lisse interne du trou empêche toute adhérence du mortier d'injection sur la paroi du trou, d'autant qu'il a été mis de l'huile sur la tige de vérin de façon à permettre une extraction plus facile. Enfin, on peut voir autant de fissurations avec trous injectés qu'avec trous non injectés.

Il n'empêche que les règles professionnelles prévoient d'injecter ces trous en commençant bien entendu par le bas et qu'il serait critiquable, pour non respect des règles, de ne pas le faire.

Un point important semble devoir être spécialement développé : les règles prévoient le cas où les fissures développées sur la paroi d'un silo ne sont pas préjudiciables permettant d'utiliser un coefficient de fissuration plus favorable et donc d'économiser de l'acier. Paradoxalement, certains constructeurs, tout en adoptant cette hypothèse, omettent de prévoir sinon une étanchéité, tout au moins une imperméabilisation extérieure. A notre avis, ou bien il y a lieu de considérer qu'une micro-fissuration est préjudiciable et donc de limiter les contraintes des aciers pour éviter ou limiter les fissurations, et dans ce cas ne pas appliquer l'étanchéité ou d'imperméabilisation ; au contraire de considérer des fissurations comme non préjudiciabes, mais dans ce cas il est impératif de prévoir au moins une imperméabilisation sinon une étanchéité.

\section{ACIERS NON DÉPLIÉS}

Deux types de désordres successifs sont la conséquence d'aciers non dépliés : en forme de $Z$ et noyés dans le béton, lorsqu'on tire dessus, ils se déplient, entraînant des fissures et des désordres importants. Par ailleurs, ce dépliement des aciers peut être accompagné d'une rupture d'aciers à haute caractéristique de résistance (FE 50 ou même acier Tor). Dépliés par l'effort de traction auquel ils sont soumis, ils peuvent casser, du fait de la fragilité des aciers à haute résistance.

Il peut donc y avoir deux désordres successifs, dépliement des aciers avec fissuration du béton, puis rupture de l'acier qui a perdu ses caractéristiques de résistance d'origine.

\section{TASSEMENTS DU DALLAGE}

Ce désordre est relativement fréquent : généralement les structures des parois et cheminées sont descendues au bon sol; au contraire, le fond du silo est réalisé sur un terrain en place souvent de médiocre qualité ou même un remblai mal mis en œuvre. Il y a bien entendu toute une part de subjectivité dans l'appréciation de l'impropriété à destination de l'ouvrage. Ou ces désordres gênent l'exploitation du silo, et rendent l'ouvrage impropre à sa destination, ou ces désordres ne gênent pas l'exploitation de l'ouvrage et ne constituent pas donc des désordres au sens de l'article 1792 du Code civil : l'appréciation relève d'une certaine subjectivité. 


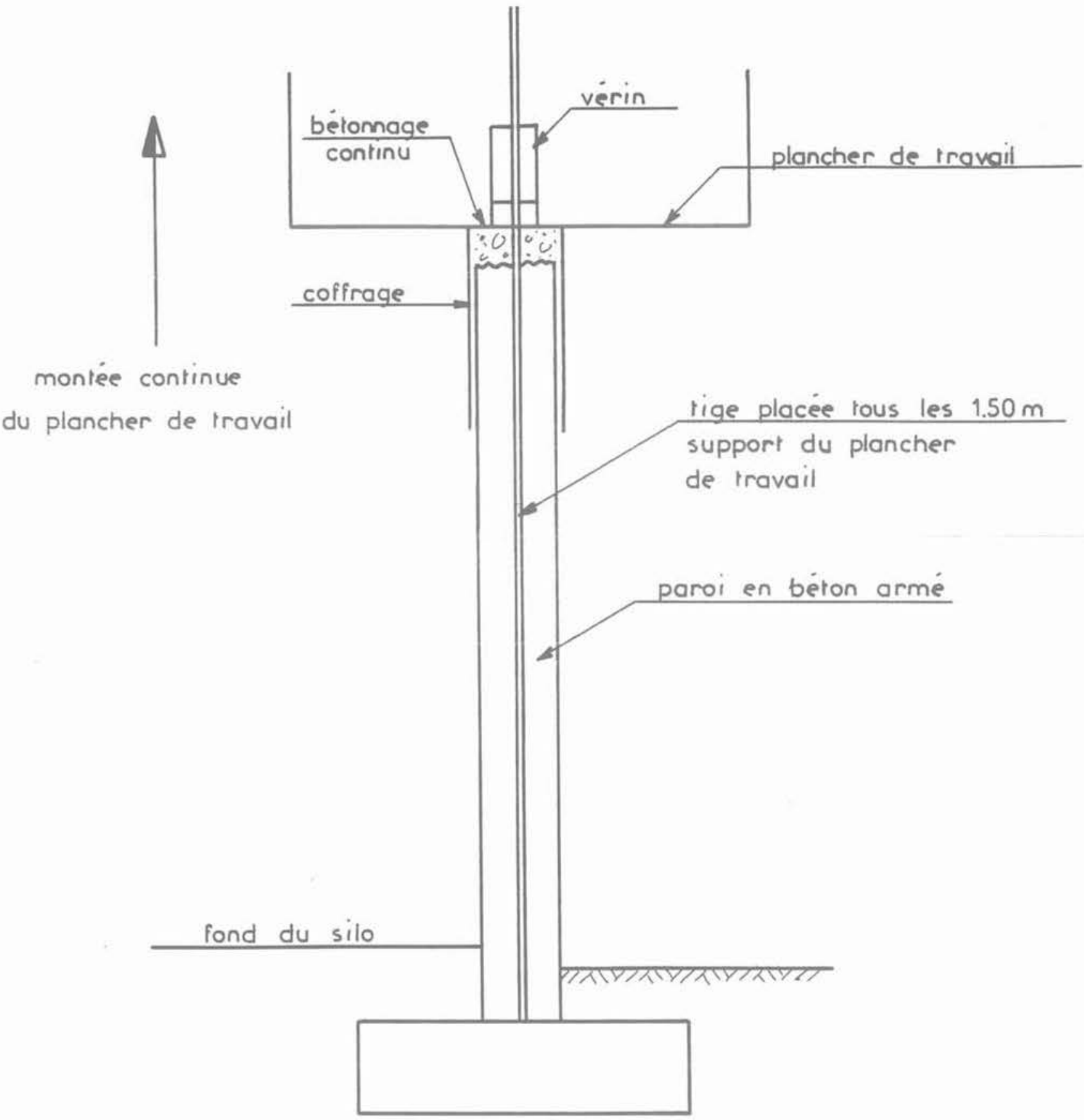

Fig. 1. - Coffrage glissant: Coupe verticale.

Fig. 1. - Sliding Schuttering: Vertical cross-section.

En tout état de cause, il y a lieu de prendre de grandes précautions dans la réalisation d'un dallage de silo dans la mesure où les charges verticales des matières ensilées entraînent des contraintes non négligeables sur le sol sous dallage.

\section{REPRISE DE LA POUSSÉE DU GRAIN PAR FROTTEMENT OU BUTÉE}

Un désordre constaté à l'occasion de la réalisation d'un silo à sucre type magasin de stockage (fig. 2), paraît intéressant à analyser.
Le désordre est le suivant : en 5-7, le joint de dilatation entre la galerie et le dallage, en 4 ans, s'est ouvert de 3 à $4 \mathrm{~cm}$; l'explication en est la suivante : la poussée du grain sur le long pan, est reprise par frottement du dallage et de la semelle de fondation du long pan sur le sol, qui est une alluvion de qualité moyenne mais n'ayant présentée aucune déformation caractéristique de tassements. Lorsque l'on remplit le silo, la poussée est équilibrée donc par le frottement du dallage sur le sol, frottement qui pour être mobilisé, nécessite un déplacement de l'ordre de $6 \mathrm{~mm}$. Il s'ensuit que, à chaque remplissage, les deux murs de long pan se déplacent chacun d'environ $6 \mathrm{~mm}$ 


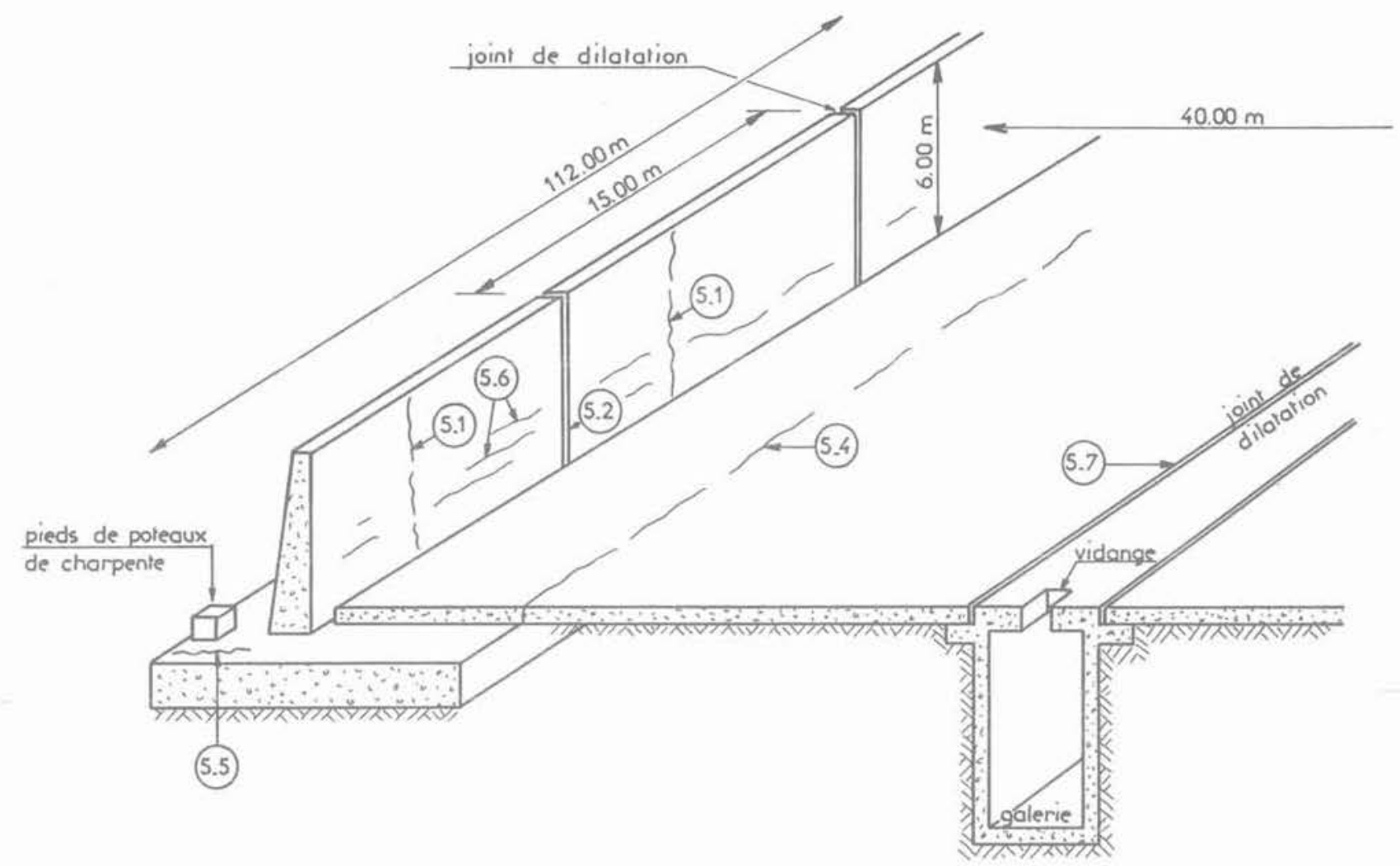

Fig. 2. - Silo à sucre. Vue axonométrique.

Fig. 2. - Sugar silo. General view.

et ne reviennent pas en arrière bien évidemment lorsqu'on vide le silo. C'est ainsi qu'en 4 ans d'exploitation correspondant à 4 vidanges, le joint, entre galerie et dallage, s'est ouvert de l'ordre de 3 à $4 \mathrm{~cm}$.

Il est bien évident que compte tenu de la nature de ce silo, ce désordre est susceptible de rendre l'ouvrage impropre à sa destination, par fissurations du dallage dans les angles, ouverture anormale des joints et risque de voir les têtes de murs de long pan, pousser sur les fermes en lamellé collé.

\section{CONCLUSIONS}

Les silos et magasins de stockage sont des ouvrages auxquels il faut apporter la plus grande attention, aussi bien à la conception qu'à la réalisation, compte tenu d'une certaine imprécision des hypothèses de poussée, de l'importance des efforts, enfin des coûts de réparation et de pertes d'exploitation généralement importants induits par des désordres. 